\title{
MONISMO, RELACIONES, Y LOS LÍMITES DE LA EXPLICACIÓN METAFÍSICA $^{1}$
}

\author{
Sebastián Briceño ${ }^{2}$
}

\begin{abstract}
Resumen: En este artículo pretendo explorar los límites de una concepción de explicación metafísica basada en el Principio de Razón Suficiente (PRS). Para ello me concentraré en una de las consecuencias contraintuitivas que supuestamente se siguen de una aplicación irrestricta del PRS, a saber: el Monismo Radical. Primero, articularé dicha concepción de explicación metafísica. Luego, explicaré cómo es que de un famoso argumento que descansa sobre el PRS (el regreso de Bradley) parece efectivamente seguirse el Monismo Radical. En seguida, argumentaré en contra de la natural reacción frente a dicho argumento, a saber: aceptar el pluralismo y las relaciones como hechos brutos últimos. Finalmente, esbozaré una versión cualificada del PRS, PRS*, que nos permite sortear el Monismo Radical sin necesidad de renunciar al contenido esencial del PRS.
\end{abstract}

Palabras clave: Principio de Razón Suficiente. Monismo. Relaciones. Regreso de Bradley. Vacuidad.

\section{INTRODUCCIÓN}

Hasta hace poco menos de trescientos años, el Principio de Razón Suficiente (PRS) guiaba las explicaciones metafísicas de muchos grandes filósofos. Pero entonces, y tras sucesivas y constantes embestidas contra la razón pura, el PRS cayó en descrédito. Hoy, tras la influencia de muchos, entre los que cabría destacar a Quine y Kripke, la metafísica, en particular la de raigambre analítica, ha restaurado la confianza en sí misma (ZIMMERMAN,

\footnotetext{
${ }^{1}$ El presente trabajo ha sido realizado en ejecución del proyecto de investigación Fondecyt-Iniciación $\mathrm{N}^{\circ} 11160724$ (Conicyt, Chile) durante una estadía de investigación en la Facultad de Filosofía de la Universidad de Oxford, Reino Unido (mayo, 2019). Agradezco a las autoridades de la Facultad las facilidades que me dieron para desarrollarlo y, en particular, a Gonzalo Rodríguez-Pereyra, por las discusiones que generosamente mantuvo conmigo mientras lo hacía. Asimismo, agradezco las observaciones de dos árbitros anónimos de esta revista y de los participantes del II Workshop Metafísica: Debates Recientes (Universidad de Chile, octubre, 2019).
}

2 Universidad de Santiago de Chile (USACH), Santiago - Chile. (iD) https://orcid.org/0000-00033040-4869 E-mail: jsbricen@gmail.com

https://doi.org/10.1590/0101-3173.2021.v44n1.29.p385

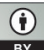

This is an open-access article distributed under the terms of the Creative Commons Attribution License. 
2004). Y, probablemente por esto mismo, el PRS ha vuelto a ser objeto de consideración favorable, explícitamente en los trabajos de Dasgupta (2016), Della Rocca $(2003,2008,2010,2012)$ y Pruss (2006), e implícitamente en la creciente y abundante literatura en torno a fundación metafísica o grounding, esto es, el tipo de explicación o determinación metafísica que intentan capturar expresiones tales como "[...] los eventos mentales existen en virtud de los eventos físicos" o "[...] las propiedades disposicionales se encuentran totalmente fundadas en las propiedades categóricas." (BLISS; TROGDON, 2016; FINE, 2001, 2012; ROSEN, 2010, 2015; SCHAFFER, 2009).

En este artículo pretendo explorar los límites de una concepción de explicación metafísica basada en el PRS. Para ello me concentraré en una de las consecuencias contraintuitivas que supuestamente se siguen de una aplicación irrestricta del PRS, a saber: el Monismo Radical (MR). En la sección 1 articularé, sin defender, tal concepción de explicación metafísica. En la sección 2 explicaré cómo es que de un famoso argumento que descansa sobre el PRS parece seguirse efectivamente el MR. Se suele sostener que, precisamente porque el PRS trae como consecuencia necesaria algo tan contraintuitivo como el MR, contaríamos con una buena razón para rechazar el PRS y aceptar en cambio pluralismo y relaciones entre esas distintas cosas como hechos brutos que constituyen, últimamente, la realidad. Así, para estos objetores, el argumento a favor del MR sería más bien una reducción al absurdo del PRS mismo. En la sección 3 argumentaré en contra de esta posición. En la última sección haré ciertas consideraciones finales donde esbozaré una versión cualificada del PRS, el PRS*, que nos permite sortear el MR y, de paso, salvar el contenido esencial del PRS del PRS mismo.

\section{EXPLICACIÓN METAFísICA Y PRS}

Frente a los hechos (cosas, estados de cosas, etc.) tenemos tres alternativas: ningún hecho es explicable; todo hecho es explicable; o algunos hechos son explicables y otros no. Y ante cualquier hecho explicable tenemos dos alternativas: explicarlo hasta cierto punto, parcialmente, aparentemente, relativamente; o explicarlo últimamente, totalmente, realmente, absolutamente.

El metafísico ideal busca explicaciones últimas de todo (BRADLEY, 1930, p. 1; BLANSHARD, 1984, p. 225). 
¿En qué consiste explicar todo? El metafísico ideal no deja trabajo inconcluso y no recurre a razones prudenciales para abandonar su investigación. Para el metafísico ideal, en principio, no hay ningún hecho inexplicable, bruto, arbitrario o primitivo. Es decir, el metafísico ideal parece acercarse al metafísico que adhiere a una versión particularmente fuerte e irrestricta del PRS. Parménides, Aquino, Spinoza, Leibniz, Bradley, por nombrar solo a algunos, se acercaron mucho a este ideal. Spinoza en particular pareció estructurar todo su sistema exclusivamente sobre el PRS (DELLA ROCCA, 2008), y abrazó una versión particularmente fuerte e irrestricta del mismo, según la cual "para cada cosa debe asignarse una causa, $o$ razón, tanto para su existencia como su no existencia" (SPINOZA, E1p11d2). Obviamente, el metafísico guiado por el PRS confía en que es posible cuantificar sobre absolutamente todo, y que el PRS aplica a todo ese rango y cae dentro de ese rango también (sobre la viabilidad de esta ambición, ver PRIEST, 2002). Todo tipo de entidad (objeto, propiedad, estado de cosas, evento, proposición, etc.), actual o posible, existente o no existente, incluido el PRS mismo, se supone, es explicable. No hay nada, no hay ningún hecho (objeto, estado de cosas, etc.), que sea inexplicable (bruto, arbitrario, primitivo, etc.), pues nada queda fuera del alcance del PRS.

¿En qué consiste explicar algo? Explicar algo es hacerlo claro a la razón, hacerlo inteligible. Esto típicamente se hace desplegando la naturaleza de la cosa. Es decir, una explicación de algo, digamos de X, supone ir más allá del hecho bruto, de la identidad bruta, que X es X. Explicar es "[...] meterse entre una cosa y sus propiedades de modo de permitir que la propiedad arroje luz sobre la cosa." (DELLA ROCCA, 2012, p. 8). Al explicar una cosa uno la hace inteligible explicitando las relaciones entre la cosa y su interior (entre la cosa y sus partes, su estructura, sus propiedades), o entre la cosa y su exterior (entre la cosa y otras cosas, sus partes, sus estructuras, sus propiedades). Spinoza también lo vio así: "[e]sta razón, $o$ causa, debe estar o contenida en la naturaleza de la cosa, o fuera de ella" (SPINOZA, E1p11d2), de tal modo que "[1]o que no puede ser concebido a través de otro, debe ser concebido a través de sí mismo" (SPINOZA, E1a2). Es decir, la relación entre explanandum y explanans no puede ser una conexión bruta. Esto atentaría contra el PRS mismo, que rechaza hechos brutos. El tipo de explicación que el metafísico ideal busca y demanda ha de estar fundada en los conceptos implicados en ella. No puede ser un hecho bruto que trascienda tales conceptos: debe fluir con la misma necesidad que toda verdad conceptual, de modo que $\mathrm{Y}$ explica $\mathrm{X}$ en virtud de los conceptos Y y X (DELLA ROCCA 2003, p. 77). Esta 
concepción racional de explicación es distintiva del PRS: algo es explicado cuando es concebido, cuando es hecho inteligible. Y un explanandum es hecho inteligible típicamente apelando a algo distinto de sí mismo, uno o más explananda. Para los efectos de este artículo, lo que me interesa destacar aquí es que toda explicación, en tanto racional, es esencialmente relacional.

Así entonces, tal como ha argumentado Della Rocca (2003, 2008, p. 8-9), el PRS tiene dos aspectos: primero, tal como señalé más arriba, todo es explicable, no hay hechos brutos; y segundo, todo es explicable en términos de su inteligibilidad. En un slogan: ser es ser inteligible o concebible. ${ }^{3} \mathrm{Y}$ esto vale para propiedades filosóficamente tan centrales como existencia, identidad y causalidad. Así lo ejemplifica la siguiente forma de explicar existencia en términos de inteligibilidad, expresión típica del PRS en acción (DELLA ROCCA, 2003, p. 82-88; SPINOZA, E1p33): Conforme al PRS, sería un hecho bruto que tanto la existencia como la no existencia de $\mathrm{X}$ fuesen inteligibles. Pues si X fuese inteligible pero no existente, entonces la no existencia de X sería un hecho bruto. Y si X fuese ininteligible pero existente, entonces la existencia de X sería un hecho bruto. Es decir, conforme al PRS es imposible que tanto la existencia de $\mathrm{X}$ como la no existencia de $\mathrm{X}$ sean inteligibles. Por tanto, si $\mathrm{X}$ es inteligible, entonces $\mathrm{X}$ debe existir. Así, ser inteligible y existir son coextensivos. Y si son coextensivos, no hay razón por la cual no sean idénticos. Pues si fueran coextensivos pero distintos, entonces su diferencia sería un hecho bruto. Pero, conforme al PRS, diferencia e identidad numéricas tampoco son hechos brutos, sino hechos explicables en términos de discernibilidad e indiscerniblidad y, últimamente, en términos de inteligibilidad. De esta forma, una típica consecuencia del PRS se hace evidente: como no pueden haber cosas inteligibles inexistentes, posibilidad colapsa en inteligibilidad, e inteligibilidad colapsa en existencia. No hay hechos (objetos, estados de cosas, etc.) meramente posibles. Si algo es ininteligible, entonces es imposible; y si algo es inteligible, entonces es existente y, por tanto, necesariamente existente.

En consecuencia, para los efectos de este artículo podemos hacer nuestra la siguiente definición del PRS que ofrece Della Rocca: “[...] por cada cosa (objeto, estado de cosas, o lo que sea) que existe o se da, hay una explicación de su existencia, una razón de su existencia" (2010, p. 1), bajo el entendido que "una razón de su existencia" es equivalente a que la existencia de la cosa

\footnotetext{
${ }^{3}$ Para los efectos de este artículo entenderé, tal como lo hace Della Rocca (2003), que "inteligible" es sinónimo de "concebible".
} 
sea inteligible, de modo que algo existe si y solo si es inteligible, y no existe si y solo si es ininteligible.

¿Qué es explicar algo en términos últimos? Entiendo que una explicación última es una explicación metafisica guiada por el PRS.

Una explicación metafísica es una explicación constitutiva, y, por tanto, distinta, en principio, a otras explicaciones paradigmáticas, a saber: teleológicas, normativas, y causales (DASGUPTA, 2016, p. 380-381). Una explicación metafísica responde a un por qué $X$ que es mejor frasear como en virtud de qué $X$, o en qué consiste $X$, o qué es realmente $X$. Así, por ejemplo, si preguntamos por qué hay una huelga, una explicación causal respondería algo así: porque los dueños despidieron a varios trabajadores. Una explicación teleológica respondería algo así: porque los trabajadores buscan revertir la decisión de los empleadores. Y una explicación normativa respondería algo así: porque es el deber de todo trabajador unirse a otros trabajadores y hacer uso de la huelga si ella es necesaria para corregir una injusticia. Una explicación constitutiva, en cambio, espera decirnos en virtud de qué existe una huelga, o en qué consiste una huelga, o qué es realmente una huelga. Es decir, una explicación constitutiva respondería algo así: porque los trabajadores de la fábrica se rehúsan a trabajar, tienen bloqueada la entrada de la fábrica, y gritan y alzan carteles demandando la reincorporación de sus compañeros. En virtud de estos hechos es que hay una huelga.

Ahora, una explicación constitutiva guiada por el PRS no se conformará con cualquier hecho invocado como explicación, pues no tolera ningún hecho bruto. Buscará determinar en qué consiste, últimamente, una huelga. Una explicación constitutiva última $Z$ es una explicación que satisface de tal manera al intelecto que la pregunta en virtud de qué $Z$ ni siquiera puede plantearse con sentido frente a tal cosa. Este $\mathrm{Z}$ debe aparecer como algo "autoexplicatorio" sin ser un hecho bruto. Spinoza nuevamente da un ejemplo de lo que podría contar como la razón última por la cual, por ejemplo, algo no existe: "[...] la propia naturaleza de un círculo cuadrado indica la razón por la cual no existe, viz. porque encierra una contradicción [...]" (SPINOZA, E1p11d). Es decir, la razón de la inexistencia de un círculo cuadrado es, como señalé antes, porque se trata de una imposibilidad conceptual: su existencia es ininteligible y, por tanto, imposible.

Entiendo que la explicación metafísica guiada por el PRS es prioritaria a los otros tipos paradigmáticos de explicación, que la presuponen 
al menos tácitamente. Si una persona curiosa recibe, por ejemplo, una explicación causal de la muerte de alguien, naturalmente preguntará lo que todo metafísico preguntaría en su lugar: en qué consiste, últimamente, la muerte de alguien; en qué consiste, últimamente, que algo cause algo. Así, la explicación causal (o teleológica, o normativa) parece presuponer una explicación constitutiva, y una explicación constitutiva parece presuponer una explicación constitutiva última.

Como se trata de una explicación guiada por el PRS es una explicación en términos de inteligibilidad. La explicación constitutiva en general espera tener la misma fuerza que una necesidad conceptual. Si la pregunta es en virtud de qué $X$, el metafísico buscará dar con un $\mathrm{Z}$ tal que el que $\mathrm{Z}$ sea el caso determina metafísicamente que $X$ sea el caso con la misma fuerza con que la existencia de un hombre no casado determina la existencia de un soltero. Y la explicación constitutiva última en particular pretende dar con hechos últimos tales que, por un lado, no tenga sentido explicarlos, y que, por otro lado, expliquen todo el resto con la misma fuerza que una necesidad conceptual.

Lo anterior parece indicar que la explicación metafísica guiada por el PRS no es más que explicación en términos de fundación metafísica o grounding, tal como lo sugiere la abundante literatura que ha surgido en torno a esta forma básica de determinación metafísica (BLISS; TROGDON, 2016; FINE, 2001, 2012; ROSEN, 2010, 2015; SCHAFFER, 2009) y tal como lo han hecho ver algunos adherentes al PRS (DASGUPTA, 2016; DELLA ROCCA, 2014; MELAMED; LIN, 2018). No tengo problemas con entender que la explicación metafísica guiada por el PRS sea entendida como explicación en términos de grounding. Pero esto nos obliga a distanciarnos de la concepción estándar de grounding. Porque esta última suele entender, dogmáticamente, que grounding es una relación que: (i) Está bien fundada en hechos "fundamentales". (ii) Relaciona solo hechos. (iii) Impone un orden parcial estricto sobre su dominio. (iv) Es primitiva. Pero, en directa oposición a las afirmaciones anteriores, el PRS nos obliga a: (i) Sostener que los hechos XX invocados como grounds últimos no pueden ser simplemente hechos ante los cuales la pregunta en virtud de qué los $X X$ siga teniendo sentido. Pues esto equivaldría a aceptar hechos brutos y simplemente bautizarlos como "fundamentales". Tales hechos han de tener un status radicalmente distinto al resto de los hechos, de modo que sean efectivamente merecedores de la etiqueta "autoexplicatorios". Dasgupta (2016), por ejemplo, diferencia cualitativamente entre hechos aptos para ser fundados que tienen fundamento 
(hechos substantivos), hechos aptos para ser fundados que no tienen fundamento (hechos fundamentales), y hechos no aptos para ser fundados (hechos autónomos). Solo estos últimos hechos serían autoexplicatorios. La literatura estándar en torno a grounding, en cambio, no hace esta distinción: simplemente declara que las cadenas de grounding han de terminar en algún punto, sin considerar si acaso la pregunta en virtud de qué los $X X$ hace todavía sentido en dicho punto. (ii) No debemos restringir los relata de la relación a hechos sin más, pues, conforme al PRS, toda cosa (objeto, estado de cosas, o lo que sea) es explicable, y no solo los hechos en sentido estricto. (iii) Debemos renunciar a la idea de que grounding imponga un orden parcial estricto sobre su dominio, pues hay suficientes contraejemplos que ponen en cuestión la idea de que grounding sea asimétrica, irreflexiva y transitiva (RODRIGUEZPEREYRA, 2015). En particular, no parece haber razón para excluir de la idea de explicación metafísica a las identificaciones o definiciones reales, tales como Agua es $\mathrm{H}_{2} \mathrm{O}$, sin más, pues ellas son explicatorias aunque no sean asimétricas ni irreflexivas (DORR, 2016; WILSON, 2014). Y no solo eso: para el metafísico que adhiere al PRS muchas veces las identidades y las esencias no son "autoexplicatorias" (pace DASGUPTA, 2016), pues son explicables en términos de su discernibilidad e inteligibilidad/concebibilidad. Así, de hecho, lo entendió Spinoza (MELAMED; LIN 2018, \$2). (iv) Tal como lo exige el PRS, no podemos aceptar grounding como un hecho bruto sin más. En primer lugar, no parece haber razón para postular entidades fundadas cuando sus fundamentos, se supone, hacen todo el trabajo explicatorio (DELLA ROCCA, 2014). En segundo lugar, porque grounding no exhibe un carácter ostensiblemente autoexplicatorio. La abundante discusión sobre la naturaleza de grounding es prueba suficiente de lo anterior. En particular, no es claro qué explica que la relación de grounding sea explicatoria de ciertos hechos, esto es, no es claro cómo es que grounding relaciona sus relata (ver sección 2).

Por lo anterior, prefiero abandonar la expresión "grounding", y, en cambio, usar expresiones más neutrales, tales como " $\mathrm{X}$ es/existe en virtud de Y”, particularmente porque no pretendo excluir a las definiciones reales o identificaciones del ámbito de la explicación metafísica.

En principio, simpatizo con la idea de explicación metafísica guiada por el PRS en los términos expuestos, especialmente con la articulación que le ha dado Della Rocca $(2003,2008,2010,2012)$. No me queda otra alternativa. Primero, porque la alternativa según la cual todo es un hecho bruto, me parece, al menos en principio, extremadamente implausible, y dudo que sea 
digna de ser abrazada sin antes explicar (i!) en qué consiste precisamente y cuáles son sus implicancias. (Como veremos en la sección final, hay una forma de cualificar esta alternativa que la vuelve plausible e interesante.) Segundo, porque la posición intermedia, según la cual hay hechos explicables y hechos inexplicables, me parece altamente inestable, especialmente porque, como veremos en la sección 3, no parece haber una línea divisoria que, por razones de principio, sea capaz de separar lo explicable de lo no explicable. Puede ser un camino pragmáticamente aconsejable, pero un metafísico no deja trabajo inconcluso por razones puramente prudenciales. Finalmente, una vez aceptado que todo es explicable, esto es, el PRS, parece que no queda alternativa que optar por explicar todo últimamente. Pues una explicación parcial o aparente es solo otro hecho explicable con el que el metafísico se topa en el camino. Y si es explicable, entonces debe explicarlo. (Aunque también cualificaré esta alternativa en la sección final.)

Por cierto, es dudoso que el metafísico ideal haya tenido expresión histórica. Como veremos a continuación, ni siquiera Spinoza siguió el PRS hasta sus últimas consecuencias. En cierto sentido, Bradley llegó más lejos, pero también dejó mucho sin explicar y consideró varias cosas inexplicables (BRADLEY, 1935, p. 650). Esto es natural. Somos humanos. Podemos abrazar un ideal y no estar a la altura de ese ideal. ¿Pero qué explica el rechazo del ideal? ¿Qué explica el rechazo del PRS hoy? Aquí solo puedo esbozar algunas razones de tal rechazo. Supongo que en parte se debe al espíritu de los tiempos (post-Hume, post-Kant, post-Moore, post-Russell, post-Wittgenstein); en parte, porque parecen no haber buenos argumentos directos en favor del PRS mismo (no es claro que el PRS sea explicable (i!)); y, finalmente, tal vez lo más importante, porque las consecuencias que parecen seguirse de una aplicación irrestricta del PRS despiertan naturalmente la sospecha de que el PRS no es una premisa digna de ser abrazada en primer lugar (BENNETT, 1984, cap. 5; RUSSELL, 1910; VAN INWAGEN, 2015, cap. 7). Tales consecuencias serían, por nombrar las más relevantes: identidad de los indiscernibles; necesitarismo radical; inexistencia de libre albedrío; colapso de lo posible, lo existente y lo concebible; existencia necesaria de un ser autoexplicatorio (Dios); y un monismo más radical que el de Spinoza: MR. No puedo hacerme cargo de todas estas consecuencias aquí. Solo me concentraré en explorar la última de ellas. 


\section{Del PRS al MONISMo RADicAl}

Como adelanté, aunque pocos filósofos parecen acercarse a Spinoza en su amplia aplicación del PRS, hay un filósofo que a mi juicio lo aplicó, al menos con respecto a ciertas cuestiones, en términos más profundos. $\mathrm{Me}$ refiero a Bradley. Bradley aplicó el PRS a la idea misma de que una cosa podía hacerse inteligible en términos de sus relaciones con sus partes, su estructura, sus propiedades, o sus relaciones con otras cosas (y sus partes, sus estructuras, sus propiedades), pues él aplicó el PRS a la idea misma de relación y la consideró ininteligible. Es el argumento de Bradley, guiado por el PRS, el que parece llevarnos al MR. Pues Spinoza entendió que el Uno era inteligible por medio de sus múltiples atributos. Pero esto significa que substancia y atributos no son idénticos, y que, en consecuencia, hay una relación, al menos de diferencia, entre ellos. Pero si las relaciones son ininteligibles, entonces el monismo de Spinoza es ininteligible, y ha de ser sustituido por el MR.

Bradley esgrimió argumentos en contra de la idea misma de relación y del pluralismo que ella conlleva en diversos pasajes de su obra (BRADLEY, 1914, cap. IX; 1922, p. 96; 1930, caps. 2 y 3, y Apéndice B; 1935). De hecho, se trata más bien de una familia de argumentos. He aquí una reconstrucción de uno de ellos:

(i) Si últimamente hay dos o más términos, entonces últimamente hay una o más relaciones.

(ii) Las relaciones son últimamente inexplicables, pues son últimamente ininteligibles.

(iii) $\mathrm{Si}$ las relaciones son últimamente inexplicables, pues son últimamente ininteligibles, entonces últimamente no existen.

(iv) Luego, últimamente, hay un solo término, el Absoluto.

Todo parece descansar en las premisas (ii) y (iii) del argumento. La premisa (iii) es simplemente el PRS, que Bradley asume, al menos tácitamente, como un principio guía de su metafísica, en términos tanto destructivos como constructivos. En varias ocasiones Bradley se lamenta de no poder dar cuenta racional de las relaciones, de no poder hacerlas inteligibles y, por tanto, de considerarlas últimamente inexplicables. Y de su ininteligibilidad última infiere entonces su irrealidad o inexistencia última. 
¿Pero por qué, según Bradley, las relaciones serían inexplicables? Porque precisamente no hay una explicación sobre cómo es que una relación relaciona sus términos. Y claramente una relación de hecho relacionando no es un hecho de aquellos que se presentan al intelecto como autoexplicatorios o como no aptos para ser explicados. El PRS nos obliga a buscar una explicación. El problema es que, cuando intentamos explicar cómo es que una relación relaciona sus términos, no encontramos una explicación.

En una de sus versiones (BRADLEY, 1930, cap. 2), el argumento de Bradley pone en cuestión la inteligiblidad de las relaciones por la vía de mostrar la ininteligibilidad de la relación entre una cosa y su naturaleza, es decir, poniendo en jaque la idea misma de una identidad explicatoria o definición real. El dilema que subyace al argumento de Bradley es parecido al que encontramos en las reflexiones sobre la identidad que Wittgenstein hace en el Tractatus (WITTGENSTEIN, 5.5301, 5.5303, 5.534, 5.53), o en la paradoja del análisis que parece afectar las definiciones reales realizadas por Moore (LANGFORD, 1968). Así prefiere expresarlo Bradley: "Si predicas lo que es diferente, adscribes al sujeto lo que no es; y si predicas lo que no es diferente, no dices nada en absoluto". (BRADLEY, 1930, p. 17). Los detalles de esta primera versión los he abordado en otro lugar (BRICEÑO, 2016), así es que ahora me concentraré en una segunda versión. En esta segunda versión (BRADLEY, 1930, cap. 3; 1935, p. 657-658), el argumento de Bradley se extiende en general en contra de la idea de cualidades en relación, y lo cierto es que sus razonamientos son aplicables a la idea misma de varios términos en relación, cualquiera sea la categoría ontológica de esos términos y de esa relación, pues basta con que se trate de entidades numéricamente distintas. El punto central de Bradley es que no parece haber salida: una relación está obligada a hacer dos cosas que parecen imposibles de combinar: unir dos términos y mantener su diversidad. Sucede que si las tomamos sin sus términos, las relaciones son ininteligibles; y si las tomamos con sus términos, también son ininteligibles.

Veamos. ¿Qué explica que Rxy? Si tomamos a $\mathrm{R}$ como algo distinto, separado e independiente de $x$ e $y$, esto es, si tomamos a $\mathrm{R}$ como algo totalmente externo a los términos, como algo que no altera su naturaleza, entonces evidentemente $\mathrm{R}$ no explica $\mathrm{R} x y$, pues si $\mathrm{R}$ no es nada para sus términos, entonces $x$ e $y$ realmente no están relacionados por R. Para Bradley (1930, p. 27), 
[...] una relación que de alguna manera precipita términos que no estaban allí antes, o una relación que se puede mantener en algún sentido sin términos, [...], es realmente una frase sin sentido. Es, a mi juicio, una falsa abstracción, y algo que se contradice abiertamente a sí mismo.

Es decir, una relación externa es algo totalmente arbitrario, bruto, inexplicable. Una relación no puede ser una entidad libremente flotante: “[...] debe haber una razón por la que esto y eso aparecen juntos”, “[...] un cómo y un por qué" de toda particular relación (BRADLEY, 1930, p. 517, 521).

Pero, por otro lado, si apelamos a los términos de Rxy, es decir, si consideramos a $\mathrm{R}$ como siendo algo para $x$ e $y$, como una relación en algún sentido fundada en sus términos, entonces no es explicable cómo es que de hecho los relaciona. Pues si $\mathrm{R}$ es también algo en sí, algo distinto de sus términos, entonces se hace necesario explicar cómo es que se relaciona con sus términos. Así, si la relación

[...] es algo para [sus términos], entonces claramente requeriremos de una nueva relación de conexión. Pues la relación difícilmente puede ser el mero adjetivo de uno o ambos de sus términos... Y, siendo algo ella misma, si ella misma no está en relación con sus términos, ¿̇de qué inteligible manera logrará ser algo para ellos? Pero aquí nuevamente nos vemos arrastrados hacia el remolino de un proceso desesperado, puesto que estamos obligados a avanzar, encontrando nuevas relaciones sin fin. (BRADLEY, 1930, p. 2728. Énfasis en el original)

¿Por qué Bradley descarta que la relación pueda ser "el mero adjetivo de uno o ambos de sus términos"? Que no es el adjetivo de uno de ellos, es claro, pues si fuese así, no solo sería arbitrario que fuese de uno de ellos y no del otro, sino que obviamente no los relacionaría. ¿Pero acaso no es lo propio de una relación ser una propiedad de adicidad superior a 1, esto es, ser "el mero adjetivo de...ambos de sus términos"? Bradley no cree que esto último pueda ser defendido sin incurrir en una petición de principio, es decir, sin presuponer aquello que está en disputa: el poder de las relaciones para relacionar sus términos. Desde luego, si la relación es tomada como una propiedad a distribuir entre sus términos tomados individualmente, entonces nuevamente no parece haber relación entre ellos. Pero si la relación es tomada como una propiedad de sus términos tomados colectivamente, como un todo, entonces ya estamos presuponiendo que los términos en cuestión están relacionados, que coexisten de cierta manera, es decir, no es claro que estemos 
frente a dos términos que no estuviesen ya relacionados-contra nuestra suposición inicial. La relación ahora parece ser una propiedad monádica que predicamos de un todo preexistente. Y si es así, el problema vuelve a surgir, pues ¿qué es la inherencia de una propiedad monádica en un todo si no una relación entre un todo y una propiedad monádica? En términos técnicos contemporáneos, el dilema es el siguiente: para que una relación relacione, es condición necesaria que esté instanciada en todos sus términos tomados colectivamente (una relación no instanciada evidentemente no relaciona: no es nada para sus términos). ¿Pero cómo es que instanciación puede explicar el que una relación relacione sus términos, si instanciación también es una relación que supuestamente relaciona sus términos?

Esquemáticamente:

¿Qué explica que Rxy?

No: R

No: $\mathrm{R} x$

No: Ry

No: $\mathrm{R} x, \mathrm{R} y$

No: Rxy

¿R'?

¿Pero qué explica que R'(Rxy)?

Es en este punto donde obviamente nos enfrentamos al famoso regreso de Bradley: explicar cómo una relación relaciona sus términos nos lleva a postular relaciones infinitamente, sin que ninguna de ellas explique cómo es que de hecho relaciona sus términos. Lo cierto es que parecen haber otras alternativas, aunque igualmente insatisfactorias: (i) un regreso circular de relaciones: R' relaciona $x$ e $y$ en virtud de $\mathrm{R}, \mathrm{y}$ R relaciona $x$ e $y$ en virtud de R', ad infinitum; o (ii) un hecho bruto: $\mathrm{R}$ relaciona $x$ e $y$ (o R' relaciona $\mathrm{R}, x$ e $y$ ) porque los relaciona, esto es, usando la expresión de MacBride (2011, p. 170), porque tiene "virtus relativa". En suma, cuando intentamos explicar cómo es que una relación relaciona sus términos enfrentamos una suerte de trilema de Agripa: o un regreso infinito, o un regreso circular, o un hecho bruto. 
Nótese que es irrelevante si acaso la relación es más o menos externa, o más o menos interna. Es la idea misma de la realidad última de cualquier relación la que está en cuestión (BRADLEY, 1935, p. 643-646, 667-668). Cierto, la relación externa, en tanto presuntamente independiente de la naturaleza intrínseca de los relata, es obviamente más sospechosa. Y cierto, la relación interna, en tanto constitutiva o dependiente de sus términos, parece más cercana a la realidad. Pero estas tienen una naturaleza inestable: en tanto relaciones, afirman la distinción de sus relata; en tanto internas, la niegan (BRADLEY, 1914, p. 239-240, 1935, p. 643-645; CANDLISH, 2007, p. 159-161; HYLTON, 1990, p. 54-55); y lo cierto es que solo trasladan el problema de cómo es que una relación relaciona varios términos al interior de los términos, pues, tratándose de relaciones internas,

[los términos] deben ser, y deben también estar relacionad[o]s. Pero luego hay una diversidad que cae al interior de cada [término]. Cada un[o] tiene un doble carácter, como soportando y siendo constituid[o] por la relación. Puede ser tomado a la vez como condición y resultado, y la cuestión es cómo puede combinar esta variedad. (BRADLEY, 1930, p. 26. Énfasis en el original)

Nótese también que, a pesar de las maniobras de algunos (e.g., MAURIN, 2010; SIMONS, 1994), es irrelevante si acaso la relación en cuestión es un tropo o un universal. El argumento tampoco depende de que tomemos a las relaciones como substancias, como otros suponen (e.g., CANDLISH, 2007; HYLTON, 1990; WOLLHEIM, 1959). Si las relaciones son algo así como substancias, obviamente el regreso se gatilla; pero si son algo más etéreo, también: "Si tomas la conexión como una cosa sólida, debes mostrar, y no puedes mostrar, cómo es que los otros sólidos se le unen. Y, si la tomas como una suerte de medium o atmósfera insubstancial, no será ya una conexión." (BRADLEY, 1930, p. 28). Lo cierto es que, como he argumentado en otro lugar, para que se gatille el regreso todo lo que se requiere es que busquemos unir, relacionar, entidades numéricamente distintas (BRICEÑO, 2016).

Nótese que términos y relaciones no son totalmente falsos o inexistentes; simplemente no son últimamente verdaderos, existentes o reales. Todo el aparataje conceptual de términos y relaciones es incluso necesario para efectos prácticos. Es necesario admitir la validez relativa de distinciones que presuponen relaciones, como las distinciones esencial/accidental, interna/ 
externa, sujeto/objeto, etc. Pero, últimamente, el aparataje conceptual de términos y relaciones, no sería más que una abstracción (violenta, viciosa, defectuosa) de una totalidad que trasciende términos y relaciones; una expresión inadecuada de una unidad supra-relacional: el Absoluto (BRADLEY, 1930, p. 125, 522, 667-668; CANDLISH, 2007, caps. 2 y 7; HYLTON, 1990, p. 55).

Nótese que inherencia o instanciación también está expuesta al argumento. Bradley fue, al menos en este sentido, más allá que Spinoza en su aplicación del PRS. Si inherencia es la relación entre una cosa y sus propiedades, entonces tal relación probaría la falsedad de cualquier MR. Pero Bradley fue enfático: "Simple inherencia, si es relacional, es a mi juicio autocontradictoria" (BRADLEY, 1935, p. 649; BRADLEY, 1935, p. 650, 671).

Y nótese, finalmente, que Russell, aunque se opuso a la conclusión de Bradley, estaba igualmente convencido de la relevancia e implicancias de la cuestión en disputa:

La cuestión de las relaciones es una de las más importantes que surge en filosofía, pues muchos otros problemas descansan sobre ella: monismo y pluralismo; la cuestión de si acaso hay algo totalmente verdadero excepto toda la verdad, o totalmente real excepto toda la realidad; idealismo y realismo, en algunas de sus formas; quizá la existencia misma de la filosofía como una disciplina distinta de la ciencia [...]. (RUSSELL, 1956, p. 333)

Asimismo, en una de sus varias polémicas, Russell también dio con el diagnóstico correcto sobre la premisa subyacente al argumento de Bradley en contra de las relaciones:

Esta opinión [i.e., que Rxy no puede simplemente ser un hecho "fortuito"] me parece que descansa sobre alguna ley de razón suficiente, algún deseo de mostrar que toda verdad es "necesaria". Tiendo a pensar que buena parte de mi desacuerdo con el Sr. Bradley descansa sobre la noción de "necesidad" [...] me parece que fundamentalmente las verdades son meramente verdaderas de hecho, y que la búsqueda por una "razón suficiente" es errada. (RUSSELL, 1910, p. 374; énfasis agregado)

Bradley (1930, p. 29) concluye el capítulo 3 de Appearance and Reality con las siguientes palabras:

El lector que ha seguido y ha captado el principio de este capítulo, tendrá poca necesidad de gastar su tiempo en los que vienen. Habrá visto 
que nuestra experiencia, cuando es relacional, no es verdadera; y habrá condenado, casi sin una audiencia, la gran masa de los fenómenos.

Y Bradley está en lo cierto. Si su argumento es correcto, entonces toda concepción relacional de la realidad es últimamente falsa. Pues si últimamente no hay relaciones, entonces últimamente no hay causalidad, ni pluralismo, ni inherencia, ni tiempo, ni cambio, ni espacio, ni distinción entre sujeto y objeto, ni hechos que fundan otros hechos, etc. Últimamente, solo nos queda el MR. Todo esto es intuitivamente chocante. No es extrańo que Russell y Moore se hayan rebelado justamente contra una filosofía que dejaba en el suelo todo fragmento de conocimiento que forma parte del sentido común y de la ciencia. ¿Qué hacer?

\section{Pluralismo bruto, argumentos De eXPlicabiLIDAd Y PRS}

Si el MR efectivamente se sigue de una aplicación irrestricta del PRS, entonces tal vez esa consecuencia sea precisamente una razón para rechazar el PRS y abrazar la idea según la cual, últimamente, hay relaciones y pluralismo, y esto es simplemente un hecho bruto. Así, al parecer, razonan Russell (1910) y van Inwagen (2015, p. 44) cuando aceptan, contra Bradley, la existencia real o última de relaciones y de pluralismo como hechos brutos. Se trata de una intuición firme: uno podría decir que un argumento que tiene como consecuencia el MR es un mal argumento, precisamente porque el MR es intuitivamente implausible. Y el argumento de Bradley parece plausible, salvo en lo que respecta a la idea de que tiene que haber una explicación por la cual dos o más términos se encuentran R-relacionados. Y esta idea no es más que el PRS. ¿Cuál es el punto entonces de incluir esta premisa-el PRS? ¿Por qué aceptar la demanda por explicar cómo es que las relaciones relacionan? Bien podríamos decir que hay varias cosas, y que las relaciones las relacionan porque las relacionan; que la realidad, últimamente, es una pluralidad de entidades relacionadas. Punto. Este es un hecho bruto, inexplicable. Es decir, no hay razón para aceptar la premisa subyacente en el argumento monista: el PRS mismo. El pluralismo y las relaciones tendrían status mooreano.

Esta posición sería plausible si no existiera un argumento directo en favor del PRS, pues sería una intuición más fuerte operando en contra de una intuición más débil. Pero Della Rocca (2010) ha ofrecido un argumento independiente en favor del PRS, que paso a discutir a continuación. 
Los filósofos aceptan argumentos de explicabilidad y, en consecuencia, entienden que muchas demandas por explicación son legítimas. Un argumento de explicabilidad tiene la siguiente estructura:

(i) Si X no es en virtud de algo, entonces $\mathrm{X}$ es inexplicable.

(ii) Pero $\mathrm{X}$ no es inexplicable.

(iii) Luego, $\mathrm{X}$ es en virtud de algo.

Los filósofos, al aceptar muchos argumentos de explicabilidad, presuponen que un rasgo problemático del mundo es explicado por algún rasgo menos problemático; que un rasgo superficial o aparente es explicado por un rasgo más profundo o real. Así, se aceptan como legítimas las demandas de explicación cuando el valor de X es, por ejemplo: referencia, concepto, persona, modalidad, semejanza, composición, conciencia, disposiciones, causalidad, leyes de la naturaleza, cambio, conocimiento, justicia, belleza, felicidad, amor, etc. Por cierto, que la práctica totalidad de los filósofos acepte al menos algún argumento de explicabilidad no habla en favor de un determinado tipo de explicación por sobre otro. Tampoco nos habla en favor del PRS mismo, pues aceptar, por ejemplo, un argumento de explicabilidad para la causalidad es compatible con rechazar un argumento de explicabilidad para la conciencia y, por tanto, compatible con un rechazo del PRS.

Pero la aceptación de argumentos de explicabilidad locales sí introduce cierta presión intuitiva que apunta en el sentido del PRS. El filósofo que acepta algunos argumentos de explicabilidad, pero que rechaza el PRS, se ve enfrentado a la siguiente pregunta: ¿por qué razón rechaza ciertos argumentos de explicabilidad y acepta otros? Dicho filósofo podría responder diciendo que la línea que divide argumentos de explicabilidad aceptables e inaceptables es arbitraria: simplemente otro hecho bruto más entre varios. Y al hacerlo así no elude cuestión alguna. Asimismo, suponer que uno debe dar una razón para dividir buenos y malos argumentos de explicabilidad es entender que otra demanda local de explicabilidad es legítima-y no supone adherir al PRS.

¿Pero qué sucede si a ese filósofo lo enfrentamos al siguiente argumento de explicabilidad? Tal como podemos demandar una explicación de causalidad (referencia, concepto, persona, modalidad, etc.), entonces parece legítimo demandar una explicación por la existencia de las cosas. ¿En virtud de qué las cosas que existen, existen? ¿Qué explica que esto y eso sean casos de existencia? 
(i) Si la existencia de cada cosa no es en virtud de algo, entonces la existencia de cada cosa es inexplicable.

(ii) Pero la existencia de cada cosa no es inexplicable.

(iii) Luego, la existencia de cada cosa es en virtud de algo.

Es decir, a partir de la aceptación de varios argumentos de explicabilidad locales es posible generar presión intuitiva para aceptar un argumento de explicabilidad relativo a la existencia, salvo que uno pueda trazar una línea divisoria entre este caso y los otros. Pero el caso de la existencia hace ahora urgente la necesidad de trazar una línea no arbitraria, no bruta, entre argumentos de explicabilidad aceptables y no aceptables, pues la aceptación del argumento de explicabilidad para el caso de la existencia nos compromete directamente con el PRS. De hecho, demandar una explicación por la existencia de cada cosa no es otra cosa que el PRS. Este es el punto central del argumento de Della Rocca (2010).

Entonces solo parecen existir tres opciones: (i) aceptar todos los argumentos de explicabilidad; (ii) rechazar todos los argumentos de explicabilidad; o (iii) aceptar algunos y rechazar otros argumentos de explicabilidad. La opción (i) equivale a aceptar el PRS, pues aceptar todos los argumentos de explicabilidad implica aceptar el argumento de explicabilidad de la existencia, y aceptar el argumento de explicabilidad de la existencia no es otra cosa que aceptar el PRS. La opción (ii) es poco plausible, pues todos, incluso los no filósofos, somos receptivos a algún argumento de explicabilidad. (Sin embargo, diré algo más sobre esta opción en la sección final, pues creo que hay una versión interesante de ella y porque creo que es una opción más estable que (iii).) La opción (iii) obliga a trazar una línea entre argumentos de explicabilidad aceptables y no aceptables. Esta línea tiene que dejar el argumento de la explicabilidad de la existencia en el lado de los argumentos inaceptables. Esta línea ya no puede ser arbitraria, pues apelar a una línea arbitraria es apelar a un hecho bruto. Y apelar a un hecho bruto es presuponer que el PRS es falso. El problema es que presuponer tal cosa ahora si que es eludir la cuestión de fondo. Mientras que un argumento de explicabilidad local como el relativo a la causalidad podía ser rechazado apelando a una línea divisoria arbitraria sin eludir la cuestión de fondo, ahora, cuando lo que enfrentamos es directamente un argumento de explicabilidad relativo a la existencia, este no puede ser rechazado apelando a una línea divisoria 
arbitraria sin eludir la cuestión de fondo, pues el argumento de explicabilidad de la existencia es un argumento directo en favor del PRS.

He aquí un posible criterio demarcatorio no arbitrario. Nada absolutamente simple puede ser explicado. ¿Pero qué es algo absolutamente simple? Algo no condicionado ni intrínseca ni extrínsecamente, algo sin estructura interna ni externa, algo que simplemente es. ¡Pero esto se parece al Uno de Parménides! Este criterio puede ser no arbitrario, pero parece conceder el punto al monista radical. Y, como vimos, el MR parece ser consecuencia del PRS. Pero toda la idea de buscar una línea divisoria no arbitraria entre argumentos de explicabilidad aceptables y no aceptables era encontrar una línea que nos permitiera rechazar el PRS para así poder rechazar el MR.

Una vez hecho el argumento directo en favor del PRS, ¡es posible apelar a intuiciones ahora para rechazar el argumento de Bradley contra las relaciones, tal como lo hacen Russell y van Inwagen? Ya no. Ciertamente es legítimo apelar a intuiciones pluralistas para rechazar el argumento monista de Bradley, pues en este argumento el PRS figura como premisa asumida. Pero ahora, después de que el argumento de la explicabilidad de la existencia ha sido planteado, que no es otra cosa que un argumento en favor del PRS, uno no puede rechazar el argumento monista simplemente asumiendo intuitivamente que el PRS es falso. Ahora el pluralista ha de dar una razón positiva para tratar el argumento de la explicabilidad de la existencia de modo diferente, ha de trazar una línea no arbitraria que permita separar el argumento de la explicabilidad de la existencia de los demás argumentos de explicabilidad y distinguirlo como un mal argumento. La intuición pluralista ha sido directamente desafiada. El argumento de Della Rocca (2010) demuestra que la presión intuitiva de argumentos de explicabilidad locales nos ha llevado a aceptar el argumento de explicabilidad de la existencia, esto es, a aceptar el PRS mismo. Y si hay un argumento en favor del PRS, entonces es un argumento en apoyo de la premisa central del argumento monista de Bradley. Y ahora, una vez que este argumento monista es desplegado, la intuición en contra del PRS no puede ser esgrimida sin más. Sería simplemente eludir la cuestión. La carga de la prueba se ha invertido.

\section{Consideraciones FINALES: RESCATANDO AL PRS DE SÍ MISMO}

Si la aceptación intuitiva de pluralismo y relaciones como hechos brutos últimos no es viable frente a un argumento directo en favor del PRS, ¿̨ignifica 
entonces que debemos aceptar el MR de Bradley como consecuencia necesaria del PRS? Depende. ¿QQué entendemos por MR? Si seguimos a Schaffer (2010), podemos distinguir entre dos tipos básicos de monismo: Monismo Existencial (ME) y Monismo Prioritario (MP). Sin entrar en detalles, el ME sostiene que solo existe realmente una cosa, el Uno, y toda distinción al interior de él sería una mera ficción, mera ilusión o mera apariencia. En cambio, el MP acepta la real existencia de una sola cosa fundamental, el Uno, y la real existencia de muchas otras cosas. Tanto el Uno como cada una de sus partes propias son igualmente reales, pero solo el Uno es fundamental: lo múltiple está metafísicamente fundado (grounded) en el Uno.

El monismo de Bradley ciertamente no es un MP. Bradley, guiado por el PRS, rechaza la realidad última de toda relación y pluralismo. Entonces el Uno de Bradley, el Absoluto, es una unidad donde, últimamente, no hay distinciones reales. Se acerca claramente más al Uno de Parménides y, por tanto, a lo que Schaffer llama ME. ¿ Significa esto que la existencia del Uno excluye la existencia de relaciones, pluralidad, distinciones? Las distinciones de Schaffer no hacen justicia a la metafísica de Bradley, pues ni el ME ni el MP aceptan la idea de grados de existencia. Mientras el primero afirma la real existencia exclusiva del Uno, el segundo afirma la real existencia de varias cosas, aunque reconoce a una de ellas como fundamental y a las otras como fundadas. Pero, en cualquiera de las dos posiciones, algo existe o no existe, y punto. No hay términos medios. En contraste, para Bradley, las relaciones, si bien no son última o realmente existentes, sí tienen distintos grados de existencia, pues son apariencias, y las apariencias admiten distintos grados de realidad (BRADLEY, 1930 , p. 318). Sucede que a la metafísica de Bradley subyacen las siguientes ideas centrales (COLLINGWOOD, 2005, p. 238-245; WOLLHEIM, 1959, cap. 5): (i) hay una Realidad, el Absoluto, que es una unidad omnicomprensiva, consistente y supra-relacional; (ii) hay apariencias que no pueden ser últimamente reales, pues son últimamente inexplicables, ininteligibles o autocontradictorias; (iii) estas apariencias nunca son meras apariencias, pues de algún modo pertenecen y cualifican a la Realidad: la Realidad se manifiesta a través de ellas en mayor o menor grado. Es decir, si bien ninguna relación es últimamente real, pues ninguna es últimamente explicable, de ahí no se sigue que no existan sin más. Así, por ejemplo, las relaciones internas, en general, serían más cercanas a la realidad que las relaciones externas. Es esta doctrina la que hace difícil clasificar el monismo de Bradley como un ME o como un MP. Según Bradley, que existen relaciones no es algo absolutamente falso, sino relativamente falso y relativamente verdadero, pues su existencia no es 
una cuestión de todo o nada. De hecho, es el mismo PRS el que nos obliga a relativizar existencia a explicabilidad, y a entender explicabilidad en términos de inteligibilidad. Y la explicabilidad (inteligibilidad) de algo también parece ser una cuestión de grados: mientras más explicable (inteligible) algo, más existente; mientras menos explicable (inteligible), menos existente. Solo el Absoluto existiría última o realmente.

Ahora bien, ¿̇es consistente la posición de Bradley? Me parece que no. Está cruzada por una tensión interna que se sigue de esas mismas ideas centrales que subyacen a su metafísica. Dicha tensión ha sido bien identificada por Blanshard (1984, p. 225-226). Bradley dice expresamente que todo fenómeno relacional es últimamente ininteligible y, por tanto, pertenece a las apariencias. Se supone entonces que las relaciones están llamadas a ser trascendidas por esa unidad supra-relacional que es el Absoluto. ¿Pero están o no incluidas las relaciones en el Absoluto? Si están incluidas tal como las entendemos en el pensamiento racional, i.e., relacional, entonces el Absoluto no es la unidad supra-relacional que se supone que es, pues queda contaminado del carácter relacional del pensamiento racional. $\mathrm{Si}$, por otro lado, las relaciones, tal como las entendemos en el pensamiento racional (i.e., relacional), están excluidas del Absoluto, entonces el Absoluto no es omnicomprensivo como se supone que es, pues incluso las meras ficciones, meras ilusiones o meras apariencias que serían entonces las relaciones son parte de la existencia. Esta última posición equivaldría a reconocer una distinción real entre apariencia y realidad, y dejar a las relaciones del lado de las apariencias y al Absoluto del lado de la realidad supra-relacional, ambas dimensiones mediadas, seguramente, por- ¿¿qué más?!—una relación. Es decir, la existencia de un Absoluto, de algo últimamente real de carácter supra-relacional y omnicomprensivo es inconsistente con la existencia meramente aparente de relaciones: o es omnicomprensivo, y entonces no es supra-relacional; o es supra-relacional, y entonces no es omnicomprensivo.

Algunos tienden a ver en la posición de Bradley una posición parecida a la que muestra Wittgenstein al final del Tractatus (e.g., DELLA ROCCA, 2012, p. 26). El ascenso al Absoluto sería por la vía relacional, pues todo pensamiento discursivo o racional es relacional. Pero esta vía sería una escalera que debiésemos arrojar después de haber subido por ella. Llegaríamos al Absoluto, a la unidad omnicomprensiva, autoconsistente y supra-relacional, por medio de una serie de peldaños últimamente ininteligibles. La contemplación del Absoluto sería parecida a una experiencia mística, imposible de articular 
conceptualmente en la medida que toda articulación conceptual está infectada del aparataje de relaciones, distinciones y términos. Pero este camino no es satisfactorio. No es posible aceptar lo que vemos tras haber ascendido por esa escalera y, al mismo, tiempo despreciar la escalera. Si ella nos permitió subir y ver la realidad, entonces ella no es ilusoria. Si ella es ilusoria, entonces mal nos puede permitir ascender realmente (BLANSHARD, 1984, p. 224).

El punto más crítico de la posición de Bradley es que su Absoluto es inconsistente con la ruta que supuestamente nos llevó hasta él: el PRS. Pues, si todo es explicable, y la medida de la existencia de algo está dada por su explicabilidad (inteligibilidad), y toda explicación es esencialmente relacionali.e., es un meterse entre la cosa y sus propiedades e iluminar la cosa por medio de sus propiedades-, entonces el Absoluto, como unidad no relacional o supra-relacional no es explicable (inteligible). Y si no es explicable (inteligible), entonces, conforme al PRS mismo, no puede ser real, no puede existir. Es decir, pareciera ser que una aplicación irrestricta del PRS nos conduce a un MR, pero un MR no es compatible con el PRS. Enfrentamos un dilema: o nos aferramos al PRS, y entonces abandonamos o trascendemos el MR; o abrazamos el MR, y entonces abandonamos el PRS. El problema es que una aplicación irrestricta del PRS parece traer consigo una refutación del PRS mismo. Pues, según el argumento de Bradley, la idea misma de explicación, qua relacional, sería últimamente inexplicable (ininteligible) y, por tanto, últimamente inexistente. El argumento de Bradley afecta a la idea misma de explicación subyacente en el PRS, pues pone en cuestión la inteligibilidad del poder dar cuenta de una cosa en términos distintos a la cosa misma.

¿Qué queda entonces? Recordemos que según el PRS todo es explicable. Y una explicación, algo que hace inteligible algo, es inherentemente relacional. Si el PRS es nuestra guía, entonces me parece que estamos obligados a sostener lo siguiente: nada es explicable en términos últimos, porque toda explicación es inherentemente relacional, y las relaciones no son explicables en términos últimos. Y como la existencia de algo es función de su explicabilidad (inteligibilidad), entonces nada existe en términos últimos. Esto vale también para el mismo PRS. Una metafísica racional nos fuerza a optar por el cuerno que nos llama a abrazar el PRS y a abandonar o trascender el MR.

Ahora, una vez removida la idea de que algo exista en términos últimos, no es claro que la idea de grados de existencia siga teniendo sentido. ¿Más o menos existente con respecto a que? Sucede algo análogo a lo que sucede cuando, rechazando la idea del metafísico fundacionista, rechazamos la idea 
de que un objeto de tamaño medio tenga que estar últimamente fundado en átomos mereológicos o en todos maximales (BOHN, 2009; SCHAFFER, 2003, 2010). Removidas esas cotas inferiores y superiores, todo objeto aparece como igualmente respetable y real. Sí resulta claro que toda existencia parece condicionada: una cosa existe en tanto explicable por otras cosas. Por decirlo de otra forma, se supone que para Bradley todo fenómeno relacional pertenece a las apariencias porque no es últimamente inteligible o explicable. En contraste con esas apariencias está el Absoluto, la unidad omnicomprensiva, autoconsistente y supra-relacional a partir de la cual uno puede medir la cercanía o lejanía de una determinada apariencia para asignarle un mayor o menor grado de existencia. ¿Pero qué sucede después de que hemos removido el Absoluto, justamente por ser ininteligible? No solo hemos removido el punto que lograba determinar los mayores o menores grados de existencia, sino que hemos rechazado la distinción última entre apariencia y realidad, entre existencia y existencia última o real. ¿¿Significa esto que todo es aparente? Bueno, pero si no hay realidad última que haga sentido de la dicotomía, entonces no ganamos mucho usando esa palabra. Bien podríamos decir que todo es real, aunque nada es últimamente real. Una vez rechazada la distinción apariencia/realidad, o existencia/existencia-última, sucede que el PRS y la posición según la cual nada es explicable también colapsan en una sola si es que en el primer caso introducimos la cualificación "relativamente" o "hasta cierto punto", y en el segundo caso introducimos la cualificación "absolutamente" o "últimamente", de modo que podemos aceptar la siguiente definición cualificada del PRS que podemos llamar PRS*: Todo es explicable (inteligible) relativamente. En consecuencia, no existe el Absoluto, pues como la existencia de algo es función de su explicabilidad (inteligibilidad), y toda explicación es esencialmente relacional, todo tiene una existencia condicionada. Como puede apreciarse, el PRS* es compatible con la posición según la cual nada es explicable en términos últimos o absolutos, pues, como la existencia de algo es función de su explicabilidad (inteligibilidad), y toda explicación es esencialmente relacional, nada tiene una existencia incondicionada, última o absoluta. La existencia de cualquier entidad es relativa a la existencia de otra entidad.

Hemos llegado a una conclusión que Bradley calificó como "monstruosa", aunque reconoció que no sabía cómo enfrentar, pues se trata de una conclusión que niega la existencia de la unidad supra-relacional que es el Absoluto, que Bradley consideraba la realidad última o incondicionada, la precondición y el resultado de todo (BRADLEY, 1935, p. 650, 671). Pero es una conclusión que me parece del todo cercana a la que abrazó de 
buena gana Nāgārjuna, quien negó el supuesto sobre el cual Bradley operaba: que las cosas tienen una naturaleza última que explica lo que ellas son manifiestamente. Nāgārjuna abrazó una metafísica de la vacuidad (śūnyatā). Todo es y es de muchas maneras, pero nada tiene naturaleza última o real: las cosas carecen de svabhāva (GARFIELD, 1995; SIDERITS; KATSURA, 2013; WESTERHOFF, 2009). Y lo que vale para todas las cosas vale también para el PRS, que deviene, por tanto, en el PRS*. Es difícil dar una explicación de lo que esta metafísica es, precisamente porque ella también reclama para sí misma la carencia de naturaleza última. Tal como lo hace la teología negativa con Dios, la metafísica de la vacuidad solo admite definición por la vía de la negación. Así, abrazar la metafísica de la vacuidad no es sostener una suerte de idealismo, proyectivismo, nominalismo o ficcionalismo con respecto a la naturaleza última de las cosas; no es sostener que las cosas tienen, últimamente, una naturaleza simple; no es sostener que la realidad tiene una estructura última gunky o infinitamente divisible; no es sostener que la realidad, últimamente, es solo relaciones, como un estructuralista óntico lo haría; no es sostener que la realidad es, últimamente, una; no es sostener que la realidad es, últimamente, múltiple; etc. Pues la realidad carece de naturaleza última. Las cosas están vacías de naturaleza última. Y decir esto no equivale a asignarles una naturaleza última inusual. ${ }^{4}$

BRICEÑO, S. Monism, relations, and the limits of metaphysical explanation. Trans/form/ ação, Marília, v. 44, n. 1, p. 385-410, Jan./Mar., 2021.

\begin{abstract}
My aim in this paper is to explore the limits of a conception of metaphysical explanation based on the Principle of Sufficient Reason (PSR). For this purpose, I will focus on one of the alleged counter-intuitive consequences of an unrestricted application of the PSR, namely: Radical Monism. First, I will articulate such a conception of metaphysical explanation. Then, I will explain how is it that from a famous argument that rests on the PSR (i.e., Bradley's regress) Radical Monism indeed seems to follow. Right away, I will argue against the natural reaction that such argument triggers, namely: that
\end{abstract}

\footnotetext{
${ }^{4}$ Hay muchas posibles objeciones contra esta metafísica de la vacuidad, entre ellas: que la postura se ve afectada por regresos infinitos de dependencia que la harían colapsar en una suerte de nihilismo ontológico; y que la postura sería auto-refutatoria o contradictoria. Defender la metafísica de la vacuidad de tales objeciones excede con creces las pretensiones de este artículo. A pesar de no estar de acuerdo del todo con su interpretación de la vacuidad, puedo remitir al lector a PRIEST (2014, caps. 11 y 12), quien ha dado contundentes respuestas a tales cuestiones.
} 
we shall accept pluralism and relations as brute facts. Finally, I will sketch a qualified version of the PSR that allows us to avoid Radical Monism without compromising the essential content of the PSR.

Keywords: Principle of Sufficient Reason. Monism. Relations. Bradley's regress. Emptiness.

\section{REFERENCIAS}

BENNETT, J. A Study of Spinoza's Ethics. Indianapolis, IN: Hackett, 1984.

BLANSHARD, B. Bradley on relations. In: MANSER, A.; STOCK, G. The Philosophy of F. H. Bradley. Oxford: Oxford University Press, 1984. p. 211-226.

BLISS, R.; TROGDON, K. Metaphysical grounding. In: The Stanford Encyclopedia of Philosophy (Winter 2016 Edition), ed. E. Zalta, URL = Disponible en: nhttps://plato. stanford.edu/archives/win2016/entries/grounding/, 2016. Acceso: 15 jul. 2020.

BOHN, E. Must there be a top level? The Philosophical Quarterly, v. 59, p. 235, p. 193-201, 2009.

BRADLEY, F. H. Essays on Truth and Reality. Oxford: Oxford University Press, 1914.

BRADLEY, F. H. Principles of Logic. V. I. 2 ed. Oxford: Oxford University Press, 1922.

BRADLEY, F. H. Appearance and Reality. 2 ed. 9. imp. Oxford: Oxford University Press, 1930.

BRADLEY, F. H. Relations. In: Collected Essays. V. II. Oxford: Oxford University Press, 1935. p. 628-676.

BRICEÑO, S. El regreso de Bradley y el problema de la unidad-compleja: ‘̧tropos al rescate? Crítica: Revista Hispanoamericana de Filosofía, v. 48, n. 143, p. 47-75, 2016.

CANDLISH, S. The Russell/Bradley Dispute and its Significance for TwentiethCentury Philosophy. New York, NY: Palgrave Macmillan, 2007.

COLLINGWOOD, R. G. An Essay on Philosophical Method. Ed. J. Connelly y G. D’Oro. Oxford: Oxford University Press, 2005.

DASGUPTA, S. Metaphysical rationalism. Noûs, v. 50, n. 2, p. 379-418, 2016.

DELLA ROCCA, M. A Rationalist Manifesto: Spinoza and the Principle of Sufficient Reason. Philosophical Topics, v. 31, n. 1/2, p. 75-93, 2003.

DELLA ROCCA, M. Spinoza. London: Routledge, 2008.

DELLA ROCCA, M. PSR. Philosophers' Imprint, v. 10, n. 7, p. 1-13, 2010.

DELLA ROCCA, M. Rationalism, idealism, monism, and beyond. In: FÖRSTER, E. MELAMED, Y (dir.) Spinoza and German Idealism. Cambridge: Cambridge University Press, 2012. p. 5-26. 
DELLA ROCCA, M. Razing structures to the ground. Analytic Philosophy, v. 55, n. 3, p. 276-294, 2014.

DORR, C. To be F is to be G. Philosophical Perspectives, v. 30, p. 39-134, 2016.

FINE, K. The question of realism. Philosophers' Imprint, v. 1, n. 1, p. 1-30, 2001.

FINE, K. A guide to ground. In: CORREIA, F.; SCHNIEDER, B. (ed.). Metaphysical

Grounding. Cambridge: Cambridge University Press, 2012. p. 37-80.

GARFIELD, J. The Fundamental Wisdom of the Middle Way: Nāgārjuna's

Mūlamadhyamakakārikā. Oxford: Oxford University Press, 1995.

HYLTON, P. Russell, Idealism, and the Emergence of Analytical Philosophy. Oxford: Oxford University Press, 1990.

LANGFORD, C. H. Moore's notion of analysis. In: SCHILPP, P. A. (ed.) The

Philosophy of G. E. Moore. La Salle, IL: Open Court, 1968. p. 319-342.

MACBRIDE, F. Relations and truthmaking. Proceedings of the Aristotelian Society v. 111, n. 1, p. 161-179, 2011.

MAURIN, A.-S. Trope theory and the Bradley regress. Philosophy Compass, v. 7, n. 11, p. 794-807, 2010.

MELAMED, Y.; LIN, M. Principle of sufficient reason. In: The Stanford Encyclopedia of Philosophy (Spring 2018 Edition), ed. E. Zalta, Disponible en: URL = https://plato. stanford.edu/archives/spr2018/entries/sufficient-reason/, 2018. Acceso en: 15 jul. 2020.

PRIEST, G. Beyond the Limits of Thought. Oxford: Oxford University Press, 2002.

PRIEST, G. One: Being an Investigation into the Unity of Reality and of its Parts, including the Singular Object which is Nothingness. Oxford: Oxford University Press, 2014.

PRUSS, A. The Principle of Sufficient Reason: A Reassessment. Cambridge: Cambridge University Press, 2006.

RODRIGUEZ-PEREYRA, G. Grounding is not a strict order. Journal of the American Philosophical Association, v. 1, n. 3, p. 517-534, 2015.

ROSEN, G. Metaphysical dependence: grounding and reduction. In: HALE, B.; HOFFMANN, A. (ed.). Modality: Metaphysics, Logic, and Epistemology. Oxford: Oxford University Press, 2010. p. 109-135.

ROSEN, G. Real definition. Analytic Philosophy, v. 56, n. 3, p. 189-209, 2015.

RUSSELL, B. Some explanations in reply to Mr. Bradley. Mind, v. 19, n. 1, p. 373-378, 1910.

RUSSELL, B. Logical atomism. In: RUSSELL, B. Logic and Knowledge. London: George Allen and Unwin, 1956. p. 321-343. 
SCHAFFER, J. Is there a fundamental level? Noûs, v. 37, n. 3, p. 498-517, 2003.

SCHAFFER, J. On what grounds what. In: CHALMERS, D.; MANLEY, D.;

WASSERMAN, R. (ed.). Metametaphysics: New Essays on the Foundations of Ontology. Oxford: Oxford University Press, 2009. p. 347-383.

SCHAFFER, J. Monism: the priority of the whole. Philosophical Review, v. 119, n. 1, p. 31-76, 2010.

SIDERITS, M.; S. KATSURA. Nāgārjuna’s Middle Way. Somerville, MA: Wisdom, 2013.

SIMONS, P. Particulars in particular clothing: three trope theories of substance.

Philosophy and Phenomenological Research, v. 54, n. 3, p. 553-575, 1994.

SPINOZA, B. Ethics. In: CURLEY, E. (ed.) The Collected Writings of Spinoza. V. I1, Princeton: Princeton University Press, 1985.

VAN INWAGEN, P. Metaphysics. 4. ed. Boulder, CO: Westview Press, 2015.

WESTERHOFF, J. Nāgārjuna's Madhyamaka: A Philosophical Introduction. Oxford: Oxford University Press, 2009.

WILSON, J. No work for a theory of grounding. Inquiry, v. 57, p. 535-579, 2014.

WITTGENSTEIN, L. Tractatus Logico-Philosophicus. Trad. D. F. Pears y B. F. McGuiness. London Routledge, 2002.

WOLLHEIM, R. F. H. Bradley. Penguin Books, 1959.

ZIMMERMAN, D. W. Prologue: Metaphysics after the Twentieth Century. In:

ZIMMERMAN, D. W. (ed.). Oxford Studies in Metaphysics. V. 1, Oxford: Oxford University Press, 2004. pp. ix-xxii.

Recebido: 30/10/2019

Aceito: 04/7/2020 